\title{
Domestic dogs' gaze and behaviour in 2-alternative choice tasks
}

\author{
Julia Espinosa (j.espinosa@mail.utoronto.ca) \\ Liyuzhi Dong (liyuzhi.dong@mail.utoronto.ca) \\ Department of Psychology, University of Toronto, 100 St. George Street, Toronto ON M5S 3G3 Canada \\ Daphna Buchsbaum (daphna@brown.edu) \\ Cognitive, Linguistic and Psychological Sciences, Brown University, 190 Thayer Street, Providence RI 02912 USA
}

\begin{abstract}
Species such as humans rely on their excellent visual abilities to perceive and navigate the world. Dogs have co-habited with humans formillennia, yet we know little about how they gather and use visual information to guide decision-making. Across five experiments, we presented pet $\operatorname{dogs}(N=49)$ with two foods of unequal value in a 2-alternative choice task, and measured whether dogs showed preferential gazing, and whether visual attention patterns were associated with item choice. Overall, dogs looked significantly longer at the preferred (high value) food over the low value alternative. There was also evidence of item-dependent predictive gazedogs looked proportionally longer at the item they subsequently chose. Surprisingly, dogs' choice behavior was only slightly above chance, despite visual discrimination. These results suggest that dogs use visual information in the environment to inform their choice behavior, but that other factors may also contribute to their decision-making.
\end{abstract}

Keywords: Domestic dog; Visual attention; Preferential gaze; 2-alternative choice task; Preference test; Decision-making.

\section{Introduction}

Making choices about where to forage or hunt for food, whether that is at a grocery store, in a grove of fruit trees, or tracking a herd of prey animals, is critical to survival for many species. Deciding where to allocate time and effort in search of resources is highly dependent on being able to effectively gather information from the environment (Stephens, 2008). Primates are endowed with an excellent visual system, and frequently rely on visual information from their surroundings to guide their behavior and selectively choose which objects to interact with. For instance, primates visually assess item features (e.g., color, size) that signal item value to the beholder (Lucas et al., 2003). As a result, visual behavior in primates is also a good indicator of the individual's attention to the outside world and can help predict how they will interact with items in the environment (Santos \& Hauser, 1999; Teichroeb \& Chapman, 2014).

A particularly valuable measure of visual attention in primates is preferential gaze (for a review of animal visual perception see Winters et al., 2015). This visual behavior occurs when an individual allocates more visual attention to one scene or item over alternatives. It provides a measure of the individual's relative interest in the items, and suggests that they differentiate them based on visual features. In primates, it has been used as a preference marker for the item that is looked at longer, and is a predictor of item selection (Jantathai et al., 2013; Smith \& Krajbich, 2018). While preferential gazing is well documented in primates, relatively little is known about how prevalent it is in domestic dogs, a species that has coevolved with humans for millennia.

Domestic dogs inhabit a human built world designed for human visual perception, but as dichromats that sense a different color spectrum than humans, their visual abilities are structurally different from those of primates (for a review see Byosiere et al., 2018). Further, some would argue that as a species dogs are better suited to interacting with the world through olfaction as their primary sense, rather than through vision (Horowitz, 2017; Horowitz \& Franks, 2020). Irrespective of this debate, the question remains, do dogs glean useful decision-making information through visual perception and do they display visual attention markers similar to primates?

Previous investigations of dogs' visual perception have predominantly used photographs or screen-based methods (e.g., touch screens), asking dogs to visually compare representations of people, places, or things. Results show that when using pictures of real-world objects, dogs differentiate a wide range of stimuli, including animals (e.g., Range et al., 2008), shape or size differences (Byosiere et al., 2017), can categorize dogs from other species (Autier-Dérian et al., 2013), and can discriminate human faces based on familiarity (Huber et al., 2013; Mongillo et al., 2010) or emotional valence (Müller et al., 2015; Nagasawa et al., 2011).

These studies demonstrate that dogs are able to selectively allocate visual attention, however it is unclear whether the findings from impoverished 2D images (with other sensory information unavailable) generalize to a real-world environment. In addition, previous work has not examined whether dogs' visual attention predicts their actions on realworld objects. Here we examine whether dogs engage in preferential gazing in a real-world task and whether, as in humans, their gaze predicts their choice behavior.

In the present study, we address this gap by integrating looking and choice behavior measures in a simple 2alternative choice task (i.e., preference test) involving two food items of unequal value. To identify patterns of visual behavior that correlate with successful outcomes, we used two variations of the task, one that dogs solved easily and one where they were at (or very close to) chance at selecting the high value food. By examining both gaze and choice behavior, our study provides an important link between visual attention and action in a real-world, interactive context, extending the previous results observed with preferential gaze at symbolic representation of items. 

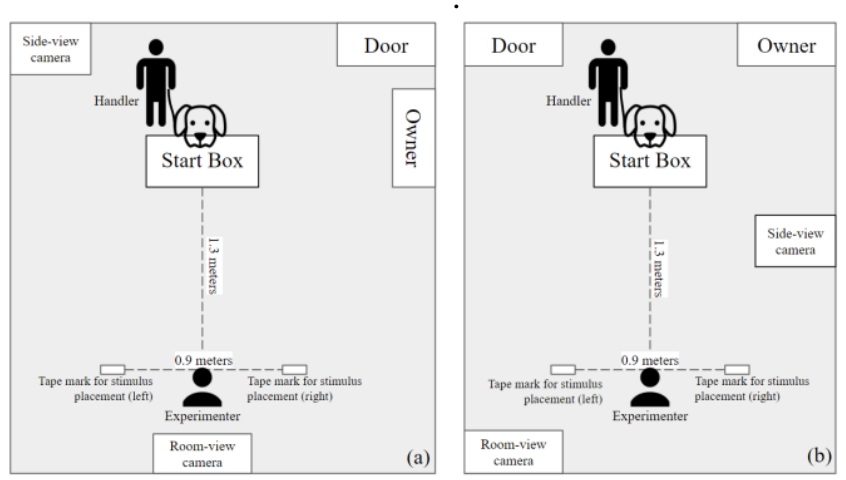

Fig. 1. Top view of testing environment for a) experiments and b) baseline and experiments

\section{Methods}

We recruited pet dogs $(N=49)$ from the city of Toronto $\left(\right.$ Male $=26 ; \mathrm{M}_{\mathrm{age}}=57.9 \mathrm{mo}$, range $\left.=6-151 \mathrm{mo}\right)$ to participate in-lab at the University of Toronto's St George campus. An additional five dogs were excluded from the final analysis for failing to complete the study $(n=2)$, or due to experimenter baiting error $(n=3)$. The dogs did not have prior experience in the lab and we did not implement any breed or training requirements for inclusion. Guardians volunteered their time and were compensated for transportation to the lab. During the study guardians remained in the room, sitting out of their dog's sightline while the task was run (Fig. 1).

\section{Materials and Procedure}

We randomly assigned dogs to a baseline condition ( $n_{\text {Baseline }}$ $=10$ ) or one of four experimental conditions $\left(n_{\mathrm{Hands}}=10\right.$; $n_{\text {Plates }}=8 ; n_{\text {Cups }}=11 ; n_{\text {Jars }}=10$ ) of the 2 -alternative choice task. In the baseline, we presented dogs with a choice between a white plate with a freeze-dried beef liver treat and a plate with nothing, to confirm that dogs would indeed consistently choose a location with a food item over an empty location (rather than exhibiting random behavior) (Fig. 1).

To motivate a preference-based choice from the dogs in the four experimental conditions, we selected two edible items of comparable size but that differed substantially in color and that were reasonably assumed to have unequal value ${ }^{1}$ to dogs. The high value items were microwaved beef hotdog slices and the low value items were dried giant white corn kernels. With similarly sized items, we controlled for the possibility that dogs made choices based on reward quantity, rather than item preference. The four experimental conditions all involved a choice between these items but presentation of the items was varied systematically. In the conditions Hands and Plates conditions, dogs watched the experimenter simultaneously present a single high and low value item either in the palms of her extended hands (arms at $45^{\circ}$ lateral

\footnotetext{
${ }^{1}$ Coding a subset of $40 \mathrm{dogs}$ for warm-up responses showed that only 16 out of 40 dogs tried to eat the corn on at least one introduction, whereas all dogs ate the hotdogs.
}

angle from the median, at dog's head height) or on two 9-inch blue plastic plates that were set on the floor $\sim 0.9 \mathrm{~m}$ apart (Fig. 1). Presenting both items simultaneously allowed the dogs to freely examine their options prior to choice. In the Cups and Jars and conditions, dogs watched as the high and low value items were presented and placed one at a time, sequentially in a counterbalanced order across trials. In the Cups condition, the experimenter dropped each item into a blue plastic cup. In the Jars condition, the experimenter showed a clear plastic jar full of either high or low value items before placing it to either the right or left. Sequential presentation encouraged the dogs to attend to each of the items individually before making a choice.

\section{Warm-up and Item Introduction}

After adjusting to the lab, dogs worked on leash with a trained handler (Fig. 1). The experimenter sat on the floor facing the dog and began each session with a simple warm-up game. The experimenter drew the dog's attention by saying "look" and extending a treat at arms-length, then placed it on the floor and verbally released the dog to approach and get the treat. Dogs that hesitated were encouraged vocally by the experimenter and the game was repeated until they approached without signs of anxiety (typically 2-4 trials).

Dogs assigned to the four experimental conditions of the 2alternative choice task were next introduced to the novel food items (corn kernel and hotdog slice) in the same manner as in the warm-up. Two trials of each item were completed, beginning with the high value item, and alternating between high and low value. This gave dogs the opportunity to learn the appearance and taste of the items prior to the choice task and established that they preferred hotdogs over corn kernels.

\section{2-Alternative Choice Test}

Dogs assigned to the baseline condition proceeded immediately to test trials following the warm-up. Dogs across all conditions worked on leash throughout warm-ups and test trials, beginning each trial facing the experimenter, and recalled after each choice to reset at the start box (Fig. 1). Both the handler and experimenter were trained to avoid cueing the dog through gaze, gesture, or vocalization to prevent influencing the dog's behavior in the task. To equalize human enhancement of the search locations, the experimenter presented both hands before simultaneously moving them laterally to the search locations and placing the treat at one and nothing at the other. A brief pause of $\sim 3$ seconds occurred between baiting and verbal release for the dog to freely observe the options. Upon making a choice, the other option was removed from access to prevent exhaustive searching. Locations were baited equally across trials, no more than twice consecutively.

The structure of the experimental test trials was nearly identical to the baseline condition, though a few changes were necessitated for the sequential presentation of Cups and 
Jars, and also the incorporation of two items into the trial demonstration. The order of showing high or low value item first was counterbalanced for sequential presentation conditions. Procedure timing and item location were consistent across all conditions.

\section{Data Scoring and Analysis}

Dogs in all conditions completed 10 test trials of the 2alternative choice task variations and were live coded by the experimenter for their approach and choice on each trial. Live coded choice results were confirmed through video recoding by a trained research assistant with $100 \%$ agreement. Choice per trial was coded based on the dog's first touch of the food item or container (i.e., cup, plate, jar, or hand) made with their muzzle or forepaw. Correct choices were scored as one, defined as touching the high value first (or in the case of baseline, the plate with the treat) and incorrect choices as zero, defined as touching the low value (or empty plate) first. Choices were summed across trials per dog, generating a possible score out of 10 .

We examined overall choice performance across all five conditions combined using a t-test (two-tailed, $\alpha=.05$ ), to determine if dogs as a group were significantly above chance at choosing the high value item. Subsequent $t$ tests looked at baseline and experimental conditions separately to determine the effect of choosing between two items (versus one item and an empty search location) on successful choice.

\section{Gaze Coding}

We video recorded dogs' gazing behavior during test from two camera angles. Videos were coded post-data collection for visual fixations by a trained coder using BORIS event logging software (Friard \& Gamba, 2016) and using an ethogram that defined visual areas of interest (AOIs) in the test environment (see OSF for full coding scheme and ethogram). Visual fixations on the AOIs were coded using the frame-by-frame function in BORIS (viewed at 60fps). Five AOIs were coded (three social ${ }^{2}$ and the two food items) as part of a broader study, but herein we will focus on the fixations to the high and low value food items that corresponded to our hypotheses about dog visual attention in a choice task. Fixations were identified from head orientation and visible eye movements (including muscles around the eyes, i.e., AU101/eyebrows). Visual fixation onset to the AOI was coded when the dog's head and/or eyes were oriented towards the AOI, and offset immediately from when the head/eyes shifted away, generating a duration in seconds.

Test trials were divided into two temporal phases: (1) observation phase where the dogs watched the experimenter present the items, and (2) approach phase where dogs walked towards one of the choices. Fixations were coded throughout, but only fixation data from the observation phase in which dogs stood facing the experimenter while she showed and

\footnotetext{
2 Visual fixations on the i) experimenter's face/torso, ii) the experimenter's empty hands (post baiting), and iii) look backs to the owner or handler were coded as measures of social referencing.
}

placed the items were used in the current analyses. This time period prior to movement forward is when dogs were evaluating the options and provided the relevant data for analyzing visual information gathering patterns that contribute to the choices. As dogs could look at each item more than once during the observation phase of a trial (e.g., looking at the high value item, then the low value, then back to the high value), the total duration of looking at the choice items per trial was calculated by summing the looking time at each item and combining the individual item durations for a total duration per item per trial. A proportion of looking time at the high versus the low value item per trial was then calculated, and proportional values are used throughout the analyses here. These looking time measures in conjunction with dogs' behavioral responses from each trial of the choice task allowed us to investigate the link between looking and acting in the real world. We focused on two aspects of visual attention: 1) preferential gazing, and 2) gaze-choice associations. They are similar measures of visual behavior, but distinct in that preferential gazing compares relative looking time between targets at a detection level, whereas gaze-choice associations (or predictive gaze) investigates the correlation between relative gaze duration at an item and item choice. By examining these two facets we aim to understand how dogs use visual information in the context of item evaluation and choice behavior.

\section{Gaze Analysis}

To explore gaze patterns in dogs, we used two series of linear mixed effects models, one testing preferential gaze at the high value item, and the other testing the predictive effect of gaze associated with choice. Both series of models included random intercepts per dog and trials nested within dog. To test for evidence of preferential gaze at the high value item, the linear mixed model included centered proportion of looking duration (s) as the dependent variable and Experiment as a predictor. The second model series tested the association between gaze and choice and used proportion of looking duration as the dependent variable with Response (a binary factor variable coded as choose high or choose low) and Experiment as predictors. Data across all five conditions was combined for initial analyses of gaze time against chance, then baseline and experimental conditions tasks were analyzed separately to take a closer look at the effect of experimental condition on gaze behavior. Trials on which dogs did not look at either of the items (i.e., looked only at the experimenter) were excluded. All statistical tests were run in R (version 4.0.3; R Development Core Team, 2020) using the glmer and lmer functions of lme4 package (version 1.126). All data and analysis scripts are available on OSF. 


\section{Results \& Discussion}

\section{Two-alternative Choice Task Performance}

We looked at overall performance choosing the high value item in the baseline and experimental conditions separately. Dogs in the baseline condition chose the high value item significantly above chance, $\left(M_{\text {correct }}=8.3,95 \%\right.$ CI [7.04, 9.56]), $t(9)=5.91, p<.001$. Dogs in the experimental conditions were also significantly above chance on this task, $\left(M_{\text {correct }}=5.87,95 \%\right.$ CI $\left.[5.43,6.31]\right), t(38)=4.00, p<.001$, albeit at a significantly lower rate than dogs in the baseline condition, $F(1,47)=22.4, p<.001$ (Fig. 2B). Post-hoc analyses with Tukey correction showed no significant difference between the means of the experimental groups, $t \mathrm{~s}$ $<1.07, .712<p s \leq .997$ (Fig. 2D).

These results suggest that dogs were somewhat successful at choosing the high value alternative in variations of the 2alternative choice task, but also that they were succeeding at a higher rate when detecting one item than they were at differentiating two items of unequal value. Interestingly, across experimental conditions, we did not observe evidence of increased accuracy selecting the high value food over trials, $B=-0.001$, S.E. $=0.012, t(339.68)=0.04, p=.970$, suggesting that dogs did not increase their selection of high value items over the 10 trials.

\section{Preferential Gaze \& Gaze-Choice Associations}

We examined the proportion of time on each trial that dogs looked at the high and low value items to explore preferential gaze at inanimate objects in dogs-i.e., whether they looked relatively longer at an item they prefer over a non-preferred. Overall, across all five conditions, dogs gazed longer at the high value item than expected by chance, $B=0.07, S E=$ $0.02, t(47.75)=4.36, p<.001$. Omnibus ANOVA analyses indicated a significant difference in looking behavior across conditions, $F(4,43.69)=4.08, p=.007$ (Fig. 2C). To unpack the results, we performed post-hoc analyses with Tukey correction for multiple testing. Results of post-hoc analyses revealed that preferential gazing was more pronounced in the baseline compared to Cups and Jars experimental conditions, baseline vs. cups: $t(42.5)=3.35, p=.014$, baseline vs. jars: $t(42.8)=3.64, p=.006$, whereas experimental conditions were not significantly different from each other, $0.11<t$ s $<$ $1.44, p s>.607$.

We next analyzed baseline and experimental conditions separately. In the baseline condition, dogs looked proportionally longer at the high value item over the empty
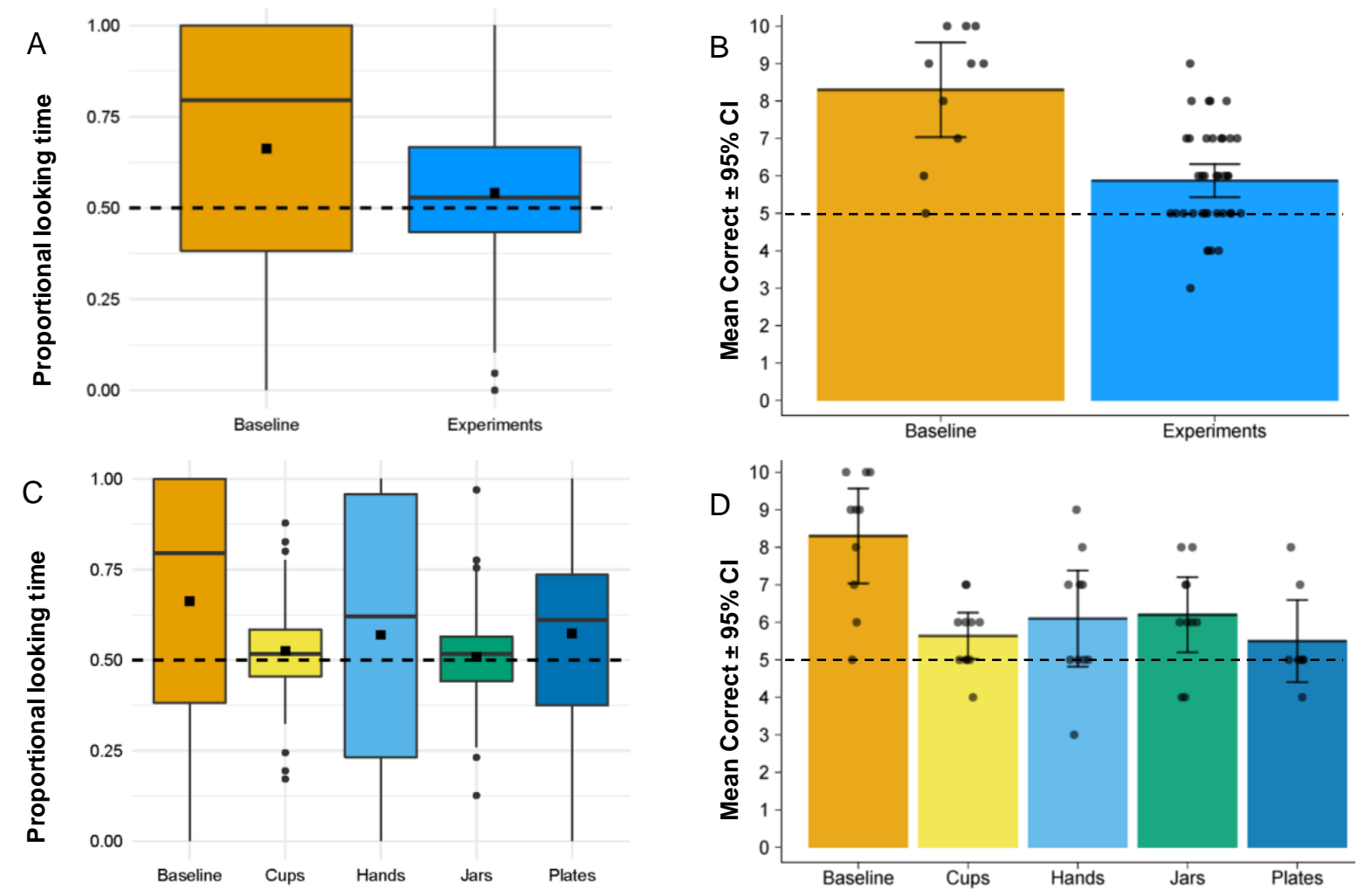

Fig. 2: A) Proportion of looking time at the high value item in baseline and combined experimental conditions. The median is represented by the solid horizontal line, the mean by the square, and the box indicates the data between upper and lower quartiles. Chance-level in all panels is indicated by the horizontal dashed line. B) Average correct response in baseline and combined experiments. Dots on bar graphs represent individual dogs' summed scores over 10 trials. C) Proportion of gaze at the high value item in separated baseline and experimental conditions. D) Average correct response in separated baseline and experimental conditions. 


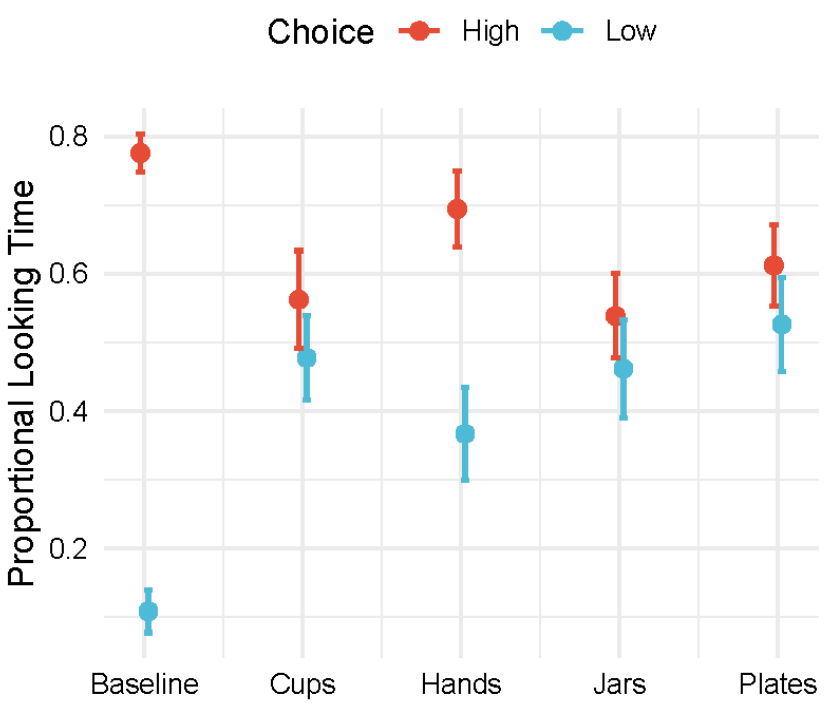

Fig. 3: Proportional looking time at high versus low value items in separate experiments. Estimated means of proportional looking time with $95 \%$ CIs.

plate, $B=0.16, S E=0.03, t(41.51)=5.46, p<.001$. Across the four experimental conditions, dogs showed a similar pattern of preferential gazing, looking proportionally longer at the high value over the low value item, $B=0.04, S E=$ 0.02, $t(37.76)=2.81, p=.008$ (Fig. 2A).

Taken together, these results suggest that dogs identify items that they prefer based on visual features, as dogs gazed proportionally longer at the high value item across all conditions. It also confirms that dogs attend to inanimate objects differentially in a real-world environment, in the context of a 2-alternative choice task, something that has been assumed in previous studies. While dogs gazed longer at the high-value item, their attention was more divided when two choice items were present (experimental conditions), than when only one option was available (baseline condition).

We applied a second series of linear mixed models to investigate gaze-choice associations - i.e., whether dogs look relatively longer at an item they subsequently select. Overall, gazing proportionally longer at an item correlated with choosing that item regardless of the item's value, $B=0.21$, $S E=0.02, t(476.99)=8.88, p<.001$, which suggests that dogs allocate more visual attention to items that they subsequently choose. We looked at experimental conditions without baseline and found the same association of longer looking at the chosen item, $B=0.14, S E=0.02, t(366.59)=$ $5.96, p<.001$. While there was an overall relationship between gaze proportion and choice, $F(4,462.45)=113.27$, $p<.001$, the choice-looking time associations varied significantly by condition (Fig. 3), Condition X Choice interaction: $F(4,462.45)=19.52, p<.001$. In particular, in the baseline condition there was a significant association between choice and looking time, $B=0.67, S E=0.06$, $t(461)=10.46, p<.001$, as well as in Hands, $B=0.33$, $S E=$
$0.05, t(468)=6.43, p<.001$, with a trend in the same direction in Cups $(p=.054)$, Plates $(p=.10)$, and Jars $(p=.11)$.

Overall, the association of looking time and item selection we observed in these experiments suggests that dogs may indeed be using visual information to make decisions in a 2alternative choice task, and that visual attention correlated with choice.

\section{General Discussion}

In a series of five, 2-alternative choice experiments that were coded for visual attention to food items of unequal value, we explored dogs' preferential gaze and gaze-choice associations to understand how they gather visual information to solve a simple task. When presented with a choice between a high value and a low value food item, dogs gazed relatively longer at the preferred (high value) item than at the non-preferred item, which is similar to patterns of human visual behavior when confronted with a analogous choice (Krajbich et al., 2010). In addition to preferential gaze, dogs demonstrated a gaze-choice association, selecting the item that they looked at proportionally longer, regardless of the item's value. Interestingly, while these findings about visual behavior suggest that dogs visually distinguish items, and may be able to use visual information to guide their choice behavior, their success at consistently picking the high over the low value item, while significantly above chance, was somewhat underwhelming. While dogs in the baseline condition chose the baited location over the empty location $87 \%$ of the time, dogs in the experimental conditions chose the preferred item over the non-preferred item approximately $59 \%$ of the time, regardless of how the items were displayed. Interestingly, this too is consistent with dogs' gaze behavior - while dogs gazed proportionally longer at the high value item, they displayed more divided visual attention in the experimental conditions, where two items were present, than in the baseline condition with just one item.

One possible explanation for why we see a lower rate of high value choices in the experimental conditions may be attributed to executive function. Previous research using simple detour and choice tasks has suggested that dogs vary in their ability to exercise inhibitory control, and that individuals may show different levels of competence depending on the problem solving situation (Bray et al., 2014). In our task, dogs may have anticipated where to search for the high value item and been unable to inhibit that action plan after observing the actual location of the items. This may also have been supported by a win-stay-lose-shift strategy, which would have prompted dogs to avoid a location based on failing to find the reward there on the immediately preceding trial. In fact, recent results have suggested that in some choice situations dog may default to just such a strategy (Byrne et al., 2020). Similarly, dogs may have identified the high value item, but nonetheless been distracted by the presence of the alternative low value choice.

Together, this could mean that while dogs visually perceived the difference between the choices, they may not 
have been able to update their approach/avoidance motor plan to incorporate new visual information about item locations. This may also be a potential explanation underlying the lack of improvement over trials in experimental conditions; while able to discriminate the items, choice strategy would have been limited by executive control.

Another possible explanation for dogs' relative difficulty in the experimental conditions could be that dogs did not visually attend to both items on every trial. Dogs may have fixated on one of the items and neglected to evaluate the other, either entirely, or for long enough to identify it. While this may be optimal if dogs noticed the high value item first, if they looked at the low value item first, dogs would have been required to reason by exclusion, and avoid the item they had fixated on in favor of approaching the unobserved option on the assumption that it was the higher value alternative. It is possible that a minimum duration threshold along with visual assessment of all options is a key for dogs to correctly discriminate the options and make successful choices. Examining the number and duration of gazes to each item is a fruitful direction for future work.

One concern might be that dogs in our task are making olfactory rather than visual choices. Even though dogs have excellent olfactory abilities, results from pet dogs suggest that they may not necessarily integrate olfactory information in 2alternative choice contexts. For instance, in an olfactory quantity discrimination task, pet dogs did not appear to differentiate large from small amounts of food based on scent (Horowitz et al., 2013), though they succeeded at visual versions of the task (Prato-Previde et al., 2008; Ward \& Smuts, 2007). Similarly, when tasked with finding a baited pot in a 3-alternative choice task at distances of one or three meters, dogs exhibited a win-stay-lose-shift strategy or random choices, suggesting that they were not using odor cues (Polgár et al., 2015). Ultimately, we think it unlikely that dogs in the current study used olfactory information to solve the task. It is reasonable to conclude that dogs were relying on visual information in this set of tasks, and the addition of the low value item presented additional discrimination challenges in experimental conditions not present in the baseline.

Another aspect of looking behavior in choice tasks that could be examined in future studies is whether social referencing to humans is correlated with choice of high or low value items. Some research has suggested that dogs are more likely to socially reference humans when they are uncertain or seeking help (Merola et al., 2012), though there is debate about the function of the behavior as a problem solving strategy, and whether it is an artifact of training, socialization, or the individual's persistence (Lazzaroni et al., 2020). Observing patterns of looking at humans when dogs are presented with multiple choice options may provide more insight into their problem-solving strategies.

The results of our work on dog gaze behavior indicate that dogs, like primates, can use visual information to solve visually-based choice tasks. Future exploration in this area should undertake to determine how gaze-choice associations in dogs may be subject stimulus salience and reward value, factors that appear to influence human attention and behavior (Blanco \& Sloutsky, 2020).

These findings underscore the importance of collecting multiple behavior measures from nonhumans. On the surface, looking only at choice results would suggest that dogs either do not have a strong preference between hotdogs and corn kernels or that they are barely able to distinguish the items. By examining looking behavior, we can determine that these low-level explanations are unlikely to be the cause of their mediocre performance, and therefore other features of the task or executive function may be impacting their behavior. Dogs are undoubtedly gifted when it comes to scent detection, but we also believe that there is a great deal of information that they perceive visually, and hope that future studies will undertake to uncover more about how dogs visually make sense of the world.

\section{Acknowledgements}

Many thanks to our participants, the CogSci anonymous reviewers for their thoughtful feedback, and the research assistants of the Computational Cognitive Development Lab for their help with data collection. This research was supported by the Natural Sciences and Engineering Research Council of Canada [2016-05552].

\section{References}

Autier-Dérian, D., Deputte, B. L., Chalvet-Monfray, K., Coulon, M., \& Mounier, L. (2013). Visual discrimination of species in dogs (Canis familiaris). Animal Cognition, 16(4), 637-651. https://doi.org/10.1007/s10071-0130600-8

Blanco, N. J., \& Sloutsky, V. M. (2020). Attentional mechanisms drive systematic exploration in young children. Cognition, 202, 104327.

https://doi.org/10.1016/j.cognition.2020.104327

Bray, E. E., MacLean, E. L., \& Hare, B. A. (2014). Context specificity of inhibitory control in dogs. Animal Cognition, 17(1), 15-31. https://doi.org/10.1007/s10071013-0633-z

Byosiere, S.-E., Chouinard, P. A., Howell, T. J., \& Bennett, P. C. (2018). What do dogs (Canis familiaris) see? A review of vision in dogs and implications for cognition research. Psychonomic Bulletin \& Review, 25(5), 17981813. https://doi.org/10.3758/s13423-017-1404-7

Byosiere, S.-E., Feng, L. C., Chouinard, P. A., Howell, T. J., \& Bennett, P. C. (2017). Relational concept learning in domestic dogs: Performance on a two-choice size discrimination task generalises to novel stimuli. Behavioural Processes, 145, 93-101. https://doi.org/10.1016/j.beproc.2017.10.009

Byrne, M., Bray, E. E., MacLean, E. L., \& Johnston, A. M. (n.d.). Evidence for Win-Stay-Lose-Shift in Puppies and Adult Dogs. 6.

Friard, O., \& Gamba, M. (2016). BORIS: A free, versatile open-source event-logging software for video/audio coding and live observations. Methods in Ecology and 
Evolution, 7(11), 1325-1330.

https://doi.org/10.1111/2041-210X.12584

Horowitz, A. (2017). Smelling themselves: Dogs investigate their own odours longer when modified in an "olfactory mirror” test. Behavioural Processes, 143, 17-24. https://doi.org/10.1016/j.beproc.2017.08.001

Horowitz, A., \& Franks, B. (2020). What smells? Gauging attention to olfaction in canine cognition research. Animal Cognition, 23(1), 11-18. https://doi.org/10.1007/s10071019-01311-z

Horowitz, A., Hecht, J., \& Dedrick, A. (2013). Smelling more or less: Investigating the olfactory experience of the domestic dog. Learning and Motivation, 44(4), 207-217. https://doi.org/10.1016/j.lmot.2013.02.002

Huber, L., Racca, A., Scaf, B., Virányi, Z., \& Range, F. (2013). Discrimination of familiar human faces in dogs (Canis familiaris). Learning and Motivation, 44(4), 258269. https://doi.org/10.1016/j.lmot.2013.04.005

Jantathai, S., Danner, L., Joechl, M., \& Dürrschmid, K. (2013). Gazing behavior, choice and color of food: Does gazing behavior predict choice? Food Research International, 54(2), 1621-1626. https://doi.org/10.1016/j.foodres.2013.09.050

Krajbich, I., Armel, C., \& Rangel, A. (2010). Visual fixations and the computation and comparison of value in simple choice. Nature Neuroscience, 13(10), 1292-1298. https://doi.org/10.1038/nn.2635

Lazarowski, L., Rogers, B., Waggoner, L. P., \& Katz, J. S. (2019). When the nose knows: Ontogenetic changes in detection dogs' (Canis familiaris) responsiveness to social and olfactory cues. Animal Behaviour, 153, 61-68. https://doi.org/10.1016/j.anbehav.2019.05.002

Lazzaroni, M., Marshall-Pescini, S., Manzenreiter, H., Gosch, S., Přribilová, L., Darc, L., McGetrick, J., \& Range, F. (2020). Why do dogs look back at the human in an impossible task? Looking back behaviour may be overinterpreted. Animal Cognition, 23(3), 427-441. https://doi.org/10.1007/s10071-020-01345-8

Lucas, P. W., Dominy, N. J., Riba-Hernandez, P., Stoner, K. E., Yamashita, N., Calderön, E. L.-, Petersen-Pereira, W., Rojas-DurÁN, Y., Salas-Pena, R., Solis-Madrigal, S., Osorio, D., \& Darvell, B. W. (2003). Evolution and function of routine trichromatic vision in primates. Evolution, 57(11), 2636-2643. https://doi.org/10.1111/j.0014-3820.2003.tb01506.x

Merola, I., Prato-Previde, E., \& Marshall-Pescini, S. (2012). Dogs' Social Referencing towards Owners and Strangers. PLoS ONE, 7(10), e47653. https://doi.org/10.1371/journal.pone.0047653

Mongillo, P., Bono, G., Regolin, L., \& Marinelli, L. (2010). Selective attention to humans in companion dogs, Canis familiaris. Animal Behaviour, 80(6), 1057-1063. https://doi.org/10.1016/j.anbehav.2010.09.014

Müller, C. A., Schmitt, K., Barber, A. L. A., \& Huber, L. (2015). Dogs Can Discriminate Emotional Expressions of Human Faces. Current Biology, 25(5), 601-605.

https://doi.org/10.1016/j.cub.2014.12.055
Nagasawa, M., Murai, K., Mogi, K., \& Kikusui, T. (2011). Dogs can discriminate human smiling faces from blank expressions. Animal Cognition, 14(4), 525-533. https://doi.org/10.1007/s10071-011-0386-5

Polgár, Z., Miklósi, Á., \& Gácsi, M. (2015). Strategies Used by Pet Dogs for Solving Olfaction-Based Problems at Various Distances. PLOS ONE, 10(7), e0131610. https://doi.org/10.1371/journal.pone.0131610

Prato-Previde, E., Marshall-Pescini, S., \& Valsecchi, P. (2007). Is your choice my choice? The owners' effect on pet dogs' (Canis lupus familiaris) performance in a food choice task. Animal Cognition, 11(1), 167-174. https://doi.org/10.1007/s10071-007-0102-7

Range, F., Aust, U., Steurer, M., \& Huber, L. (2008). Visual categorization of natural stimuli by domestic dogs. Animal Cognition, 11(2), 339-347. https://doi.org/10.1007/s10071-007-0123-2

Santos, L. R., \& Hauser, M. D. (1999). How monkeys see the eyes: Cotton-top tamarins' reaction to changes in visual attention and action. Animal Cognition, 2(3), 131139. https://doi.org/10.1007/s 100710050033

Smith, S. M., \& Krajbich, I. (2018). Attention and choice across domains. Journal of Experimental Psychology: General, 147(12), 1810-1826. https://doi.org/10.1037/xge0000482

Stephens, D. W. (2008). Decision ecology: Foraging and the ecology of animal decision making. Cognitive, AFfective, \& Behavioral Neuroscience, 8(4), 475-484.

Szetei, V., Miklósi, Á., Topál, J., \& Csányi, V. (2003). When dogs seem to lose their nose: An investigation on the use of visual and olfactory cues in communicative context between dog and owner. Applied Animal Behaviour Science, 83(2), 141-152. https://doi.org/10.1016/S0168-1591(03)00114-X

Teichroeb, J. A., \& Chapman, C. A. (2014). Sensory information and associative cues used in food detection by wild vervet monkeys. Animal Cognition, 17(3), 517528. https://doi.org/10.1007/s10071-013-0683-2

Ward, C., \& Smuts, B. B. (2006). Quantity-based judgments in the domestic dog (Canis lupus familiaris). Animal Cognition, 10(1), 71-80. https://doi.org/10.1007/s10071006-0042-7

Winters, S., Dubuc, C., \& Higham, J. P. (2015). Perspectives: The Looking Time Experimental Paradigm in Studies of Animal Visual Perception and Cognition. Ethology, 121(7), 625-640. https://doi.org/10.1111/eth.12378 